\title{
The Effect of Automated Marker Detection on in Vivo Volumetric Stent Reconstruction
}

\author{
Gert Schoonenberg ${ }^{1,3}$, Pierre Lelong ${ }^{2}$, Raoul Florent ${ }^{2}$, \\ Onno Wink ${ }^{1}$, and Bart ter Haar Romeny ${ }^{3}$ \\ ${ }^{1}$ Philips Healthcare, X-Ray Predevelopment, Best, The Netherlands \\ \{gert.schoonenberg, onno.wink\} @philips .com \\ ${ }^{2}$ Philips France, Philips Research Paris, Paris, France \\ \{pierre.lelong, raoul.florent\} @philips.com \\ 3 Technische Universiteit Eindhoven, Department of Biomedical Engineering, \\ Division Biomedical Imaging and Modeling, Eindhoven, The Netherlands \\ b.m.terhaarromeny@tue.nl
}

\begin{abstract}
New drug eluting stents are less radiopaque than bare metal stents and therefore difficult to see with conventional X-ray coronary angiography. 2D StentBoost and intravascular ultrasound (IVUS) are routinely used to evaluate stent deployment and vessel apposition during a percutaneous coronary intervention. IVUS images give cross-sectional information about the stent lumen and surrounding tissue. 2D StentBoost is a boosted angiogram sequence and visualizes the geometry of the deployed stent from a fixed viewing direction. Three-dimensional motion compensated volumetric stent reconstruction has been developed to give insight into the 3D geometry of the stent. Markers on the balloon wire are used to motion compensate cardiac rotational angiography acquisitions. In this paper we present the effect of automated marker detection on in vivo volumetric cardiac stent reconstructions. Automated or semi-automated marker detection reduces user interaction, potentially reduces total processing time, and increases detection results which leads to higher quality of stent reconstructions.
\end{abstract}

Keywords: angiography, percutaneous coronary interventions, marker detection, motion compensation, coronary stent reconstruction.

\section{Introduction}

Outcomes of percutaneous revascularization are dependent upon proper visualization of the stent. The stents' location, surrounding tissue and deployed geometry are important to check proper stent expansion. Stent expansion and deployment can be assessed using intravascular ultrasound (IVUS), quantitative coronary angiography (QCA) and 2D StentBoost [1. IVUS gives cross-sectional information about the stent expansion and surrounding tissue, however it requires additional time and equipment and subjects a patient to small but incremental risks. 2D StentBoost is a boosted angiogram sequence from a fixed gantry 
position of the C-arm system, which only allows assessing the stent expansion in one viewing direction.

We present fully automatic 3D Stent Reconstruction based on rotational Xray angiography. The resulting motion compensated volumetric reconstructions give an accurate three-dimensional assessment of the stent. This technique requires no additional equipment, need for a contrast injection, nor substantial time requirement. This imaging technique provides the operator with similar results seen with intravascular ultrasound regarding stent expansion and may assist in the confirmation of complete stent expansion. However this technique gives very limited information regarding surrounding tissue.

The method of motion compensated stent reconstruction using balloon wire markers consists of several steps. First an rotational acquisition consisting of 211 projection images is made using a calibrated C-arm X-ray system. In each projection image the balloon markers are detected using an automated marker detection algorithm. Based upon two frames, that are ideally in the same cardiac phase and are acquired 90 degrees apart, a 3D model of each marker can be generated using epi-polar geometry [2]. This static 3D model of a certain cardiac phase can be forward projected onto all the acquired images of the rotational run and used for motion correction. An affine transformation can be used to warp all the images to the specific cardiac phase of the 3D model. FDK reconstruction can be used to reconstruct the projection images into the final motion compensated volume [3].

Results on synthetic data and animal data show that stents can be reconstructed assuming the motion of the markers on the balloon wire is equal to the motion of the stent [4. Initial results of 3D Stent Reconstruction in humans using an image intensifier system [5] and a flat panel detector [6] show the feasibility in a clinical setting.

In this paper we would like to focus on automated marker detection. In a clinical setting user interaction and processing time should be reduced to an absolute minimum such that the final stent reconstruction appears to the interventional cardiologist as soon as possible. We have implemented a multi-step automated marker detection algorithm for rotational acquisitions based upon a marker couple detection algorithm which will be explained in the next section. Section 3 will show the result of our fully automatic method to generate stent reconstructions. In section 4 the results will be further discussed and section 5 concludes this paper.

\section{Methods}

In this section we will explain the various steps of the marker detection algorithm.

\subsection{Data Acquisition}

An acquisition consisting of 211 projection images (typically 1024x024 pixels of 154 micron) of the iso-centered stent is acquired during a continuous rotation (7.1 seconds) using a calibrated monoplane C-arm X-ray system for Interventional 
Cardiology (Allura Xper FD20, Philips Medical Systems, Best, The Netherlands). The patient is instructed to take a breath hold during the rotational run in order to minimize respiratory motion.

\subsection{Marker Couple Detection}

The only a priori information for the marker couple detection is that we search for two periodically moving markers in each frame of the rotational acquisition. These markers have a constant distance in 3D space, but due to foreshortening can have a varying distance in the projection images. The first step of the marker couple detection is to detect blob like structures. An example of blob detection is blob detection with automatic scale selection [7]. A marker couple selection algorithm is needed, because blob detection methods identify many potential markers. Potential marker couple pairs are identified based upon these found markers. Parameters, such as distance between the two markers, orientation and location of these markers, can be calculated for each marker pair. Adding a priori information results in temporal constraints for successive frames: the marker positions will have relatively small displacements between subsequent frames due to the high framerate $(30 \mathrm{~Hz})$ and small angular difference between successive frames (less than 1 degree) and relatively low heartrate (approximately $1 \mathrm{~Hz}$ ).

This marker couple detection algorithm will identify markers but also other blob like features in the rotational run. If the blob detector did not identify one of the two markers in a frame, the marker couple might not be able to identify a pair that fulfills the temporal, location and orientation constraints for that frame and the frame is simply skipped.

\subsection{Marker Couple Detection with Region of Interest}

The user can optionally set a region of interest (ROI) for the entire rotational run to reduce false positives in the marker detection process. Each marker in each frame has to be located within this ROI. Setting a ROI manually typically reduces the search area of the images by 75 to $92 \%$. Table 1 shows the size in precentage of the ROI compared with the total image size. The closer the stent is iso-centered towards the geometrical iso-center of the C-arm system and the smaller the patient motion (cardiac and respiratory) the smaller the ROI.

Table 1. ROI sizes

\begin{tabular}{|r|r|r|r|r|r|r|r|r|r|r|}
\hline Case ID & 1 & 2 & 3 & 4 & 5 & 6 & 7 & 8 & 9 & 10 \\
\hline ROI (as \% of total image) & 17.8 & 7.8 & 8.3 & 24.5 & 10.8 & 11.0 & 9.8 & 14.1 & 8.2 & 17.7 \\
\hline
\end{tabular}

\subsection{Marker Couple Detection with a ROI and 3D Model Clues}

To improve the marker couple detection results a second pass mechanism is implemented. The idea is that in a first pass only two frames with correctly detected markers are necessary to generate a $3 \mathrm{D}$ model that can serve as an 
aid for marker couple selection in the second pass. A 3D model of the two markers is generated based upon detection results of two frames from the first marker detection pass. This 3D model is forward projected to all the projection images and serves as a clue for the marker couple detection in the second pass. The distance between the markers and the angle of the imaginary line between the markers are reliable features for the marker couple selection. Due to the cardiac and possibly respiratory motion the location is a less reliable feature. The location of the model is used to serve as a ROI for the blob detection, whereas the orientation information is used for the final couple selection. Generation of the $3 \mathrm{D}$ model can fail when one or both of the selected images for generating the model have incorrect marker positions. The user can correct the marker positions or select frames in which the markers were identified correctly.

\subsection{Motion Compensation and Reconstruction}

Only frames in which a marker couple was identified can be motion compensated and will be reconstructed. Using frames that are not or not correctly motion compensated might result in reconstruction artifacts.

\subsection{Data}

In total 10 rotational runs of cardiac stents acquired in an adult population are used to evaluate the different marker couple detection strategies. The stents are Boston Scientific Taxus stents with various sizes.

\subsection{Analysis}

In order to compare results we define marker-couple detection sensitivity (Sens), specificity and positive predictive value (PPV) on run level:

$$
\begin{gathered}
\text { sensitivity }_{\text {run }}=\frac{T P_{\text {run }}}{T P_{\text {run }}+F N_{\text {run }}} \\
\text { specificity }_{\text {run }}=\frac{T N_{\text {run }}}{F P_{\text {run }}+T N_{\text {run }}} \\
p p v_{\text {run }}=\frac{T P_{\text {run }}}{T P_{\text {run }}+F P_{\text {run }}}
\end{gathered}
$$

We define a true positive (TP) as a frame in which both balloon markers are detected correctly, because we only motion compensate frames that have two correctly identified markers. A false positive (FP) is a frame in which one or both markers are detected incorrectly. A false negative $(\mathrm{FN})$ is a frame in which no markers where detected. A true negative (TN) would be a frame in which no markers are present and no markers are detected. The subscript run for TP, FP, FN, TN denotes the summation over all the images.

In our data true negatives do not appear because the stent is well iso-centered and therefore the markers are visible in all frames of the rotational acquisition. Therefore true negative is always zero and as a result the specificity is always zero. 


\section{Experiments and Results}

In this section we present the results of the various marker detection steps and the corresponding stent reconstructions.

\subsection{Marker Couple Detection Results}

For each marker couple detection strategy we list the TP, FP, FN, sensitivity and positive predictive value, see Table 2

Table 2. Marker detection results for various detection strategies (one pass without ROI; one pass with ROI; first pass with ROI and second pass with 3D model clues). *Strong foreshortening of the stent. 3D model was manually corrected for the second pass, due to marker switching in the first pass.

\begin{tabular}{|r|r|r|r|r|r|r|r|r|r|r|r|r|r|r|r|}
\hline & \multicolumn{9}{|c|}{ One pass without ROI } & \multicolumn{3}{|c|}{ One pass with ROI } & \multicolumn{3}{|c|}{ With 3D model clue } \\
\hline Case ID & TP & FP & FN & Sens & PPV & TP & FP & FN & Sens & PPV & TP & FP & FN & Sens & PPV \\
\hline 1 & 84 & 65 & 58 & 0.59 & 0.56 & 168 & 0 & 39 & 0.81 & 1.00 & 176 & 0 & 31 & 0.85 & 1.00 \\
$2 *$ & 23 & 20 & 164 & 0.12 & 0.53 & 86 & 72 & 49 & 0.63 & 0.54 & 94 & 1 & 112 & 0.46 & 0.99 \\
3 & 93 & 64 & 50 & 0.65 & 0.59 & 182 & 1 & 24 & 0.88 & 0.99 & 196 & 1 & 10 & 0.95 & 0.99 \\
4 & 148 & 0 & 59 & 0.71 & 1.00 & 185 & 0 & 22 & 0.89 & 1.00 & 186 & 1 & 20 & 0.90 & 0.99 \\
5 & 103 & 12 & 92 & 0.53 & 0.90 & 195 & 0 & 12 & 0.94 & 1.00 & 181 & 5 & 21 & 0.90 & 0.97 \\
6 & 120 & 54 & 33 & 0.78 & 0.69 & 201 & 0 & 6 & 0.97 & 1.00 & 166 & 10 & 31 & 0.84 & 0.94 \\
7 & 30 & 54 & 123 & 0.19 & 0.35 & 173 & 6 & 28 & 0.86 & 0.97 & 176 & 3 & 28 & 0.86 & 0.98 \\
8 & 65 & 22 & 120 & 0.35 & 0.74 & 101 & 3 & 103 & 0.50 & 0.97 & 166 & 3 & 38 & 0.81 & 0.98 \\
9 & 115 & 0 & 92 & 0.56 & 1.00 & 205 & 0 & 2 & 0.99 & 1.00 & 207 & 0 & 0 & 1.00 & 1.00 \\
10 & 95 & 20 & 92 & 0.51 & 0.83 & 136 & 17 & 54 & 0.72 & 0.89 & 182 & 10 & 15 & 0.92 & 0.95 \\
\hline Average & 87.6 & 31.1 & 88.3 & 0.50 & 0.73 & 163.2 & 9.9 & 33.9 & 0.82 & 0.94 & 173.0 & 3.4 & 30.6 & 0.85 & 0.98 \\
\hline Aver.(\%) & 42.3 & 15.0 & 42.7 & 50 & 73 & 78.8 & 4.8 & 16.4 & 82 & 94 & 83.6 & 1.6 & 14.8 & 85 & 98 \\
\hline
\end{tabular}

The difference in detections results with and without using a ROI is large. Without a ROI the average TP percentage is $42.3 \%$ and with a ROI it is $78.8 \%$. False positive detections drop from $15.0 \%$ to $4.8 \%$ and the false negative detections drop with $26.3 \%$. When using the first pass with a ROI and use that to generate a $3 \mathrm{D}$ clue for the second pass the detection results improve again. The TP percentage goes up with another $4.8 \%$, FP drop with $3.2 \%$ and FN drop with $1.6 \%$.

False positive detections are typically other structures such as other stents, sternal wires, stitches and other blob-like structures. Figure 1 shows on the left an example of a FP detection when not using a ROI. The 3D clue location of each marker is denoted in the right image as a square, the correctly detected markers are denoted with crosses.

\subsection{Stent Reconstruction Results}

For three cases the stent reconstruction results are shown in Figure 2 for each of the detection strategies. The lower row shows the detection result using the $3 \mathrm{D}$ model clue and manual corrections for the FP and FN. 

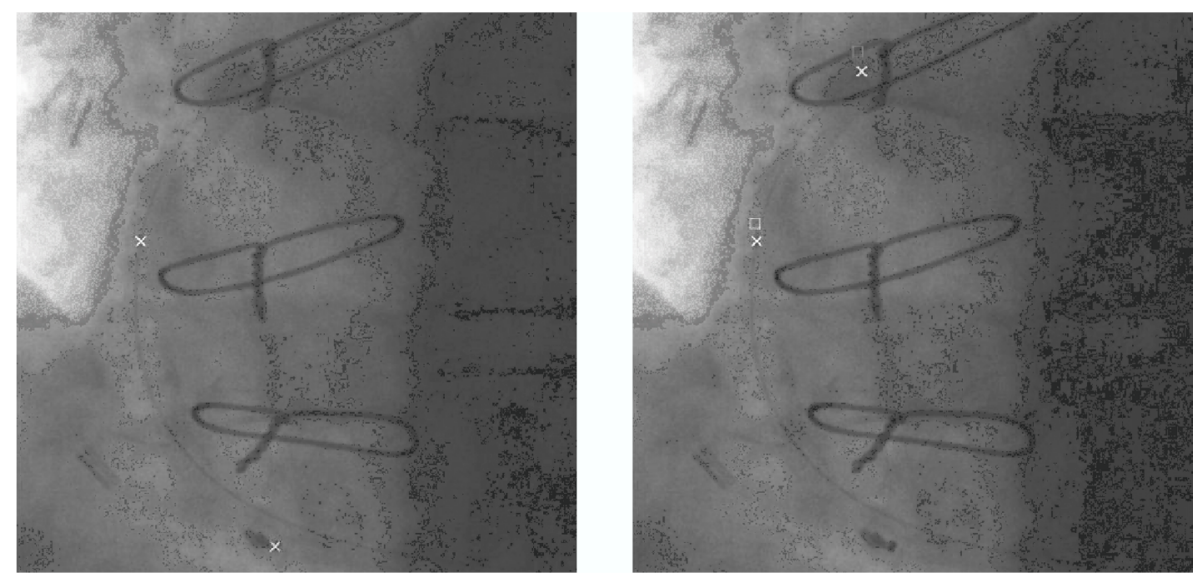

Fig. 1. Marker couple detection results. Left: a FP marker couple (detected markers denoted with crosses); a stitch is detected as marker when not using a ROI or 3D model clue. Right: correctly detected markers when using a 3D model clue (denoted with squares).

\subsection{Processing Time}

The time needed for marker detection with or without a ROI is about 65 seconds. The time to do 2 passes to improve overall marker couple detection performance is 115 seconds, this includes generating a 3D model twice. Warping all frames takes typically 20 seconds. Generating a reconstruction takes an additional 25 seconds. In total it takes 160 seconds to automatically generate a 3D stent reconstruction on a standard workstation.

\section{Discussion}

Markers are always present in all the frames of the rotational run due to proper iso-centering. However our implementation can handle true negative detections; frames in which no markers are detected will not be motion compensated or reconstructed.

Some patients have had previous interventions such as open-heart surgery or stent placement. The placed sternal wires, stitches, stents and other devices influence the marker detection results. Especially when those objects are superimposing the markers in the projection image, it is difficult to detect them. We are currently implementing marker couple interpolation techniques and 4D model based clues to improve our detection results in those challenging cases. This might also reduce the need to set a ROI manually in specific cases. 

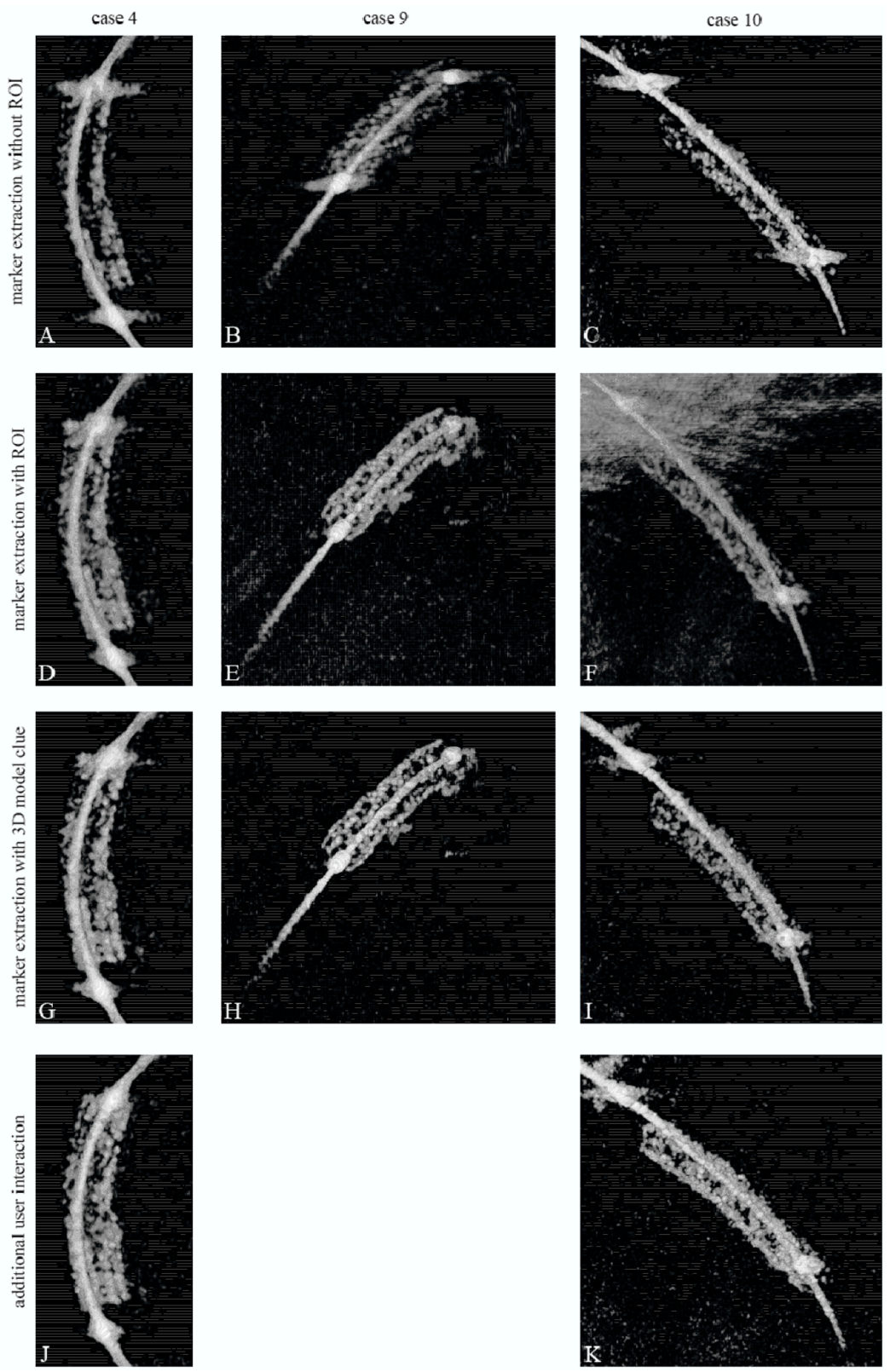

Fig. 2. Stent reconstruction results. From left to right column: case 4, 9 and 10. First row (A, B, C) motion compensation using marker extraction without ROI; second row (D, E, F) motion compensation using marker extraction with ROI; third row $(\mathrm{G}, \mathrm{H}$, I) motion compensation using 3D model clue. Case 9 achieved $100 \%$ sensitivity and needed no addition user interaction to correct missed or incorrect marker positions. The bottom row shows for case 4 and 10 the stent reconstruction $(\mathrm{J}, \mathrm{K})$ where, on average in 23 frames per case, markers are manually added. 


\section{Conclusion}

Automated 3D Stent Reconstruction using fully automatic marker detection is feasible. A flawless marker couple detection algorithm is not necessary, however better detection results will lead to better image quality. After evaluating 10 cardiac stent cases using a second pass with 3D model clues our average marker couple detection results are promising: $83.6 \%$ true positive detections, $1.6 \%$ false positive detections and $14.8 \%$ false negative detections.

Acknowledgments. The authors wish to thank the staff of the cardiac catheterization laboratories at University of Colorado at Denver and Health Sciences Center, Aurora, CO and to the patients who enrolled in this study.

\section{References}

1. Mishell, J.M., Vakharia, K.T., Portset, T.A., Yeghiazarians, Y., Michaels, A.D.: Determination of Adequate Coronary Stent Expansion Using StentBoost, a Novel Fluoroscopic Image Processing Technique. Catheter Cardio. Inte. 69, 84-93 (2007)

2. Chen, S.J., Carroll, J.D.: 3-D Reconstruction of Coronary Arterial Tree to Optimize Angiographic Visualization. IEEE Trans. Med. Imag. 19(4), 318-336 (2000)

3. Feldkamp, L., Davis, L., Kress, J.: Practical cone-beam algorithm. J. Opt. Soc. Amer. A 1(6), 612-619 (1984)

4. Perrenot, B., Vaillant, R., Prost, R., Finet, G., Douek, P., Peyrin, F.: Motion Correction for Coronary Stent Reconstruction From Rotational X-ray Projection Sequences. IEEE Trans. Med. Imag. 26(10), 1412-1423 (2007)

5. Movassaghi, B., Schaefer, D., Grass, M., et al.: 3D Reconstruction of Coronary Stents in Vivo Based on Motion Compensated X-Ray Angiograms. In: Larsen, R., Nielsen, M., Sporring, J. (eds.) MICCAI 2006. LNCS, vol. 4191, pp. 177-184. Springer, Heidelberg (2006)

6. Schaefer, D., Movassaghi, B., Grass, M., et al.: Three-dimensional reconstruction of coronary stents in vivo based on motion compensated X-ray angiography. In: Cleary, K.R., Miga, M.I. (eds.) Proceedings of SPIE, Volume 6509, Medical Imaging 2007: Visualization and Image-Guided Procedures, March 22, 2007, 65091M (2007)

7. Lindeberg, T.: Feature detection with automatic scale selection. International Journal of Computer Vision 30(2), 79-116 (1998) 